\title{
De Morgan algebras
}

\author{
PETER R, FOWLER
}

This thesis considers De Morgan algebras, an associated generalization of three valued Lukasiewicz algebras, and finally Ockham algebras.

We first consider the variety of De Morgan algebras - bounded distributive lattices with a unary operation $\sim$ which satisfies $x=\sim \sim x, \sim(x \wedge y)=\sim x \vee \sim y, \sim(x \vee y)=\sim x \wedge \sim y$ and $\sim 0=1$. There is a duality between this variety and the category of compact orderdisconnected ordered topological spaces endowed with an involution which is both a dual order-isomorphism and a homeomorphism.

The second chapter contains results for the subvariety of De Morgan algebras which is determined by the identity $(x \wedge \sim x) \wedge(y \vee \sim y)=x \wedge \sim x$; this is the variety of Kleene algebras. The nucleus is introduced as the ideal generated by the set of elements of the form $x \wedge \sim x$. Using this ideal, characterizations are obtained for the smallest congruences on a De Morgan algebra such that the quotient algebras are Kleene or Boolean. In this context a Boolean algebra is a De Morgan algebra in which $\sim$ is complementation. The characterization for Kleene algebras is used to obtain the dual spaces of the quotient algebras mentioned above which, in turn, gives a method of constructing coproducts of Kleene algebras.

At the start of Chapter 3, we describe the free De Morgan extension of a bounded distributive lattice. The remainder is devoted to De Morgan algebras which are endowed with a second unary operation $\nabla$, such that $\sim \vee \nabla x=1$ and $x \wedge \sim=\sim \wedge \nabla x$. These algebras form a generalization of three-valued Lukasiewicz algebras. We consider five related categories

Received 21 December 1981. Thesis submitted to the Flinders University of South Australia, February 1981. Degree approved: October 1981. Supervisor: Dr W.H. Cornish. 
and characterize their duals. These five categories of algebras form some of the vertices of a commuting diagram of reflections. This diagram consists of eight vertices and ten reflections. Of these reflections, four take an object to a maximal homomorphic image in a subcategory and the remaining six take an object to an essential extension. The duals of an object and one of these essential extensions are isomorphic and homeomorphic spaces. However, the order on each of the spaces is different if the extension is a proper one. The chapter is concluded with a few results which describe epimorphic extensions and the free objects, on a finite number of generators, for each of the categories.

The final chapter contains a short study on Ockham algebras, see Urquhart [3]. Ockham algebras may be considered as a generalization of De Morgan algebras, as they possess one unary operation $\sim$ which behaves as a dual lattice homomorphism.

It is first shown that any equation is equivalent to a set of inequalities, each of which is such that every variable occurs exactly twice, and the two occurrences of a variable are on the same side of the inequality sign if and only if the powers of $\sim$ are of different parities. Consequently, every subvariety of Ockham algebras can be expressed as the intersection of varieties each of which is determined by one inequality of the type mentioned above.

The above result is then used to obtain the general format of coproducts in any subvariety of Ockham algebras. The technique used is essentially the same as in Chapter 2, where we were looking at the Kleene algebras as a subvariety of De Morgan algebras.

\section{References}

[1] William H. Cornish and Peter R. Fowler, "Coproducts of De Morgan algebras", Bulz. Austral. Math. Soc. 16 (1977), 1-13.

[2] William H. Cornish and Peter R. Fowler, "Coproducts of Kleene algebras", J. Austral. Math. Soc. Ser. A 27 (1979), 209-220. 
[3] Alasdair Urquhart, "Distributive lattices with a dual homomorphic operation", Studia Logica 38 (1979), 201-209.

22 Naldera Crescent, Seacl iff Park,

South Australia 5049,

Australia. 